\title{
Role of Fusaric Acid Mycotoxin in Pathogensis Process of Tomato Wilt Disease Caused by Fusarium oxysporum
}

\author{
Mohamed E Selim ${ }^{1 *}$ and Nehad A El-Gammal ${ }^{2}$
}

${ }^{1}$ Agricultural Botany Department, Faculty of Agriculture, Menoufiya Universty, Egypt

${ }^{2}$ Plant Pathology Research Institute, Agriculture Research Center, Giza, Egypt

\begin{abstract}
Different Fusarium oxysporum isolates were tested under greenhouse conditions to determine their virulence potential toward tomato plants. Severity and development of Fusarium wilt disease was recorded 4, 6 and 8 weeks after pathogen inoculation. Chromatogram assay was conducted using HPLC to evaluate the capability of the tested isolates to produce Fusaric acid and to investigate the possible correlation between FA accumulation and Fusarium wilt disease development on tomato plants. The results showed that all tested isolates were pathogenic and varied significantly with regard to their virulence potential on tomato under the test conditions. The results showed also that all tested isolates were able to produce FA. Moreover, remarkable variation in FA accumulation was observed among the tested isolates at 4, 6 and 8 weeks post inoculation with the individual isolates. Four weeks post inoculation; the recorded data indicated that no clear correlation could be detected between FA accumulation and virulence of the tested isolates. In contrary, FA accumulation was in positive proportional relationship with virulence of the tested Fusarium isolates at 6 weeks post inoculation. Furthermore, differences in production of total secondary metabolites were observed within Fusarium isolates under in vitro conditions. These results revealed that FA could be play an important role in the development of Fusarium wilt disease at middle and last stages of tomato growth, while, it seems that no distinctive role of FA in the first stages of pathogenesis process of the tested Fusarium oxysporum isolates.
\end{abstract}

Keywords: Fusarium oxysporum; Fusaric acid; HPLC; Virulence potential

\section{Introduction}

Fusaric acid (FA) (5-butylpicolinic acid) is a host non-specific mycotoxin that is produced by various Fusarium species. The early detection of in vitro production of FA had been reported with Fusarium heterosporum Nees [1]. Since that date, the ability of other different Fusarium species to produced FA either in vitro (using substrates containing starchy material, such as corn, cassava, rice, potato, wheat etc.) or in vivo, inside susceptible host plants, had been proved and reported in many studies [2,3]. Among all Fusarium species, Fusarium oxysporum in general and its special forms lycopersici in particular was found to be able to produce the highest FA concentrations comparing to the other Fusarium species as well as to the other forma specials belonging to $F$. oxysporum species [4].

On the other hand, tomato Lycopersicum esculentum Mill is considered one of the most important vegetable crops grown for human consumption. This crop has an increasing importance nowadays specially in those countries with rapidly expanding population, e.g., Africa and Asia, where tomato production has duplicated three times since 1990 [5-7]. Unfortunately, this value crop is known as one of the most highly susceptible vegetable crops to various pathogens in general and particularly to Fusarium infection. Among all Fusarium species, Fusarium oxysporum $f$. sp. lycopersici, represented a real threat to tomato production all over the world. Thus, since it was first described in England in 1895, it was known as the most predominate and destructive causal pathogen of tomato diseases.

Fusarium oxysporum is the major causal pathogen of tomato Fusarium wilt disease. This fungus has invaded many countries in all continents i.e., North and South America, Europe, Africa, Asia, and Oceania [7]. It can attack not only tomato plants but also many other economic host plants and weeds causing severe damage [8-10].

Many studies were conducted over last decades to determine the size of FA biological activity in addition to figure out the relevance of FA in pathogenesis process of Fusarium species on their susceptible hosts. Since early nineteen's, several studies $[1,11]$ candidate this compound to play a crucial role in the pathogenesis and severity of tomato wilt symptoms caused by F. oxysporum f. sp. lycopersici Schlecht. Recently, some other researchers showed that FA is potentially toxic to animals, mice and it has several important pharmacological properties [12,13].

This present study was conducted to measure the virulence potential of 12 Egyptian Fusarium oxysporum isolates obtained from different locations. Evaluate the development of Fusarium wilt disease under green house conditions. Determine the capability of the tested Fusarium isolates to produce Fusaric acid under in vitro conditions through using High Pressure Liquid Chromatography (HPLC) analysis. Investigate the possible relation between Fusaric acid accumulation and the aggressiveness of Fusarium isolates toward tomato plants.

\section{Materials and Methods}

\section{Bioassay and virulence potential of Fusarium isolates}

Twelve pathogenic isolates belonging to Fusarium oxysporum $f_{s p}$. lycopersici were obtained from Agriculture research center, Institute of plant pathology, department of vegetable diseases, Al-Gemmaza station.

*Corresponding author: Mohamed E Selim, Agricultural Botany Department Faculty of Agriculture, Menoufiya University, Egypt, Tel: 2048-222-5446; E-mail: m_elwy76@yahoo.com

Received September 22, 2015; Accepted October 13, 2015; Published October 19,2015

Citation: Selim EM, El-Gammal NA (2015) Role of Fusaric Acid Mycotoxin in Pathogensis Process of Tomato Wilt Disease Caused by Fusarium oxysporum. J Bioprocess Biotech 5: 255 doi:10.4172/2155-9821.1000255

Copyright: ( 2015 Selim EM, et al. This is an open-access article distributed unde the terms of the Creative Commons Attribution License, which permits unrestricted use, distribution, and reproduction in any medium, provided the original author and source are credited. 
Pure cultures of Fusarium isolates were maintained on Potato Dextrose Agar (PDA) medium amended with $150 \mathrm{mg}^{-1}$ chloramphenicol. For each individual isolate, several small disks ( $1 \mathrm{~mm}$ diameter) were cut using a sterilized cork borer and stored in micro-bank tubes at $-20^{\circ} \mathrm{C}$. These initial disks were used later for preparing Fusarium inocula for further using in the next investigations.

For determining severity of tested Fusarium isolates and Fusarium wilt disease development under greenhouse conditions, three initial disks for each individual isolate were inoculated into autoclaved 500 $\mathrm{ml}$ flasks contained $200 \mathrm{ml}$ of Potato Dextrose Broth (PDB). The inoculated flasks were incubated at $27^{\circ} \mathrm{C}$ in the dark. After 2 weeks, the mycelia and spore suspension was sieved through filter papers (No.10). The number of the spores in spore suspensions was counted using a Haemocytometer slide (Thoma, Germany) and the concentration was adjusted using sterilized tap water to $1 \times 10^{5} \mathrm{spore} / \mathrm{ml}$.

Sterilized seeds of the tomato cultivar Hellfrücht (kindly provided by Bonn University, INRES-Institute, Germany) which is susceptible to Fusarium-wilt disease were sown separately in plastic pots containing $1 \mathrm{~kg}$ sterilized sand:soil mixture $(1: 1 \mathrm{w} / \mathrm{w})$. After seed germination (approximately 6 days after sowing), Fusarium inoculum of each individual isolate was inoculated at rate of $3 \times 10^{5}$ spore/pot and five replicates were treated with each individual isolate.

Disease incidence and disease severity were measured 4, 6 and 8 weeks post Fusarium inoculation according to Grattidge and O'Brien [14] based on 0 to 4 visual scale where:

$$
\begin{aligned}
& 0=(0-24 \%) \text { of leaves yellowed and wilted } \\
& 1=(25-49 \%) \text { of leaves yellowed and wilted } \\
& 2=(50-74 \%) \text { of leaves yellowed and wilted } \\
& 3=(75-99 \%) \text { of leaves yellowed and wilted } \\
& 4=(100 \%) \text { dead plant }
\end{aligned}
$$

$\%$ of disease severity was determined according to Vakalounakis and Fragkiadakis [15] using the following formula:

$$
\text { Disease severity } \%=\frac{\sum \text { scale } \times \text { number of plants inf ected }}{\text { Highest scale } \times \text { total number of plants }} \times 100
$$

\section{Fusaric acid accumulation}

The chemical detection of Fusaric acid (FA) was conducted on HPLC apparatus, Agilent Technologies 1262 Infinity system, preceded by an Eclipse plus $\mathrm{C} 18$ reverse-phase guard column $(4.6 \times 10.0 \mathrm{~mm}, 3.5$ $\mu \mathrm{m})$. The HPLC system consisted of a 1260 Quat Pump unit, Agilent Technologies 1260 diode array detector, fluorescence array detector, and a 1260 auto sampler, which were controlled by ChemoStation for LC 3D system. FA detection was achieved according to the method described by Notz et al. [16] with some modifications. However, before samples were injected, the column had been equilibrated with solvent A [ $(25 \%$ methanol $(\mathrm{v} / \mathrm{v})+0.03 \%$ TFA $(\mathrm{v} / \mathrm{v})+10 \%$ acetonitrile $(\mathrm{v} / \mathrm{v})]$. After injection, the samples were eluted at a flow rate of $1 \mathrm{ml} /$ min using an isocratic flow rate of $100 \%$ solvent $\mathrm{A}$ for 2 min and a linear gradient from $100 \%$ solvent A to $100 \%$ solvent B [Absolute methanol+0.3\% TFA (v/v)] in 13 min followed by an isocratic flow of $100 \%$ solvent B for $5 \mathrm{~min}$. For FA harvesting, pure cultures of Fusarium isolates were obtained as described above with virulence bioassay. The chromatogram investigation of the accumulated metabolic compounds and FA was achieved at 4, 6 and 8 weeks post inoculation with each individual isolate. A culture filtrate of each sample was centrifuged at $14,000 \times \mathrm{g}$ for $10 \mathrm{~min}$ to remove fungal debris. An equal volume of methanol was added to the obtained supernatant and $50 \mu \mathrm{l}$ of this mixture was injected onto the HPLC reverse phase column. Fusaric acid standard (Sigma-aldrich, kindly provided by Prof. Dr. Alexander Schouten, Bonn University, INRES-Plant Protection, Germany) was injected under the same conditions. Spectral analysis was conducted to compare the detected peaks within similar retention times in all samples with spectral pattern of FA standard (Figure 1). Fusaric acid accumulation was determined by measuring the area under the curve at $270 \mathrm{~nm}$ (optimal wavelength) with the diode array detector.

\section{Statistical analysis}

Data were statistically analyzed according to standard analysis of variance by a one-way ANOVA with Stat graphics (Statistical Graphics, Rockville, MD, USA) software; Variance homogeneity for all treatments was confirmed by the Bartlett test. Tukey's test was used to compare differences between different treatments if the F value was significant. Statistical differences referred to in the text were significant at $(\mathrm{P}<0.05)$ as given in the Figures [17].

\section{Results}

Pathogenicity test was conducted under greenhouse conditions to determine the virulence of twelve Fusarium oxysporum isolates infecting the susceptible tomato plants (cv. Hellfrücht). Also, developing of Fusarium wilt disease was evaluated through measuring the dramatic changes in percentage of disease severity at 4,6 and 8 weeks post inoculation. The obtained results revealed that all tested Fusarium isolates were pathogenic of tomato plants and causing visual wilt disease under in vivo conditions. Also, there were clear significant variances among the tested isolates in their virulence (Figure 2). At 4 weeks post inoculation, the highest percentage of disease severity, $72.21 \%, 66.38 \%, 55.21 \%, 46.65 \%, 37.77 \%$, and $36.78 \%$ was recorded with isolates 12, 3, 4, 2, 10 and 1, respectively (Figure 2). At 6 weeks post inoculation, the results showed that the isolates number 12, 2, 3, 1 and 4 resulted in the highest percentage of disease severity expressed as $89.50 \%, 76.30 \%, 72.98 \%, 64.34 \%$ and $57.48 \%$, respectively (Figure 2 ). Similar results were observed at 8 weeks post inoculation. Thus, it could be concluded that the highest disease severity i.e., $98.27 \%, 94.15 \%$ and $92.48 \%$ was recorded with isolates 12, 2 and 3 respectively (Figure 2).

Moreover, the bio-chemical assay using HPLC chromatogram analysis was achieved to determine the in vitro accumulation of Fusaric acid and the concentrations of total secondary metabolites. Data were collected four weeks, six weeks and eight weeks after the Fusarium isolates inoculation. The obtained results illustrated in Figure 3 show that all tested isolates were able to produce Fusaric acid mycotoxin under in vitro conditions. Moreover, remarkable diversity was observed

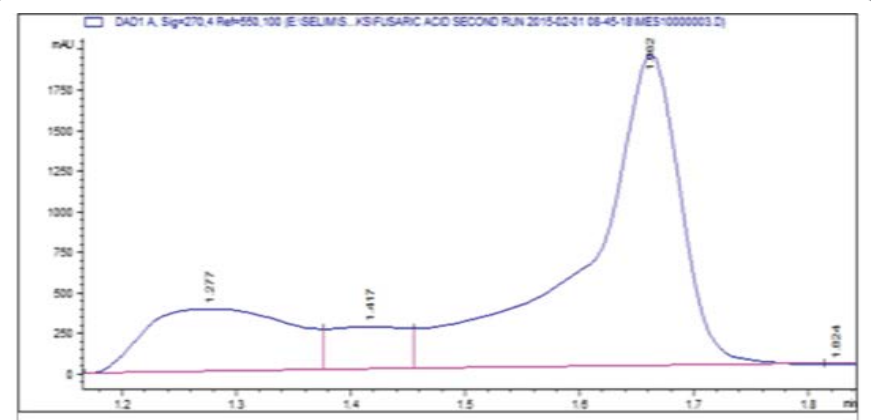

Figure 1: Spectral analysis of the peaks detected at similar retention times (at $270 \mathrm{~nm}$ wavelength) within the tested Fusarium isolates. 


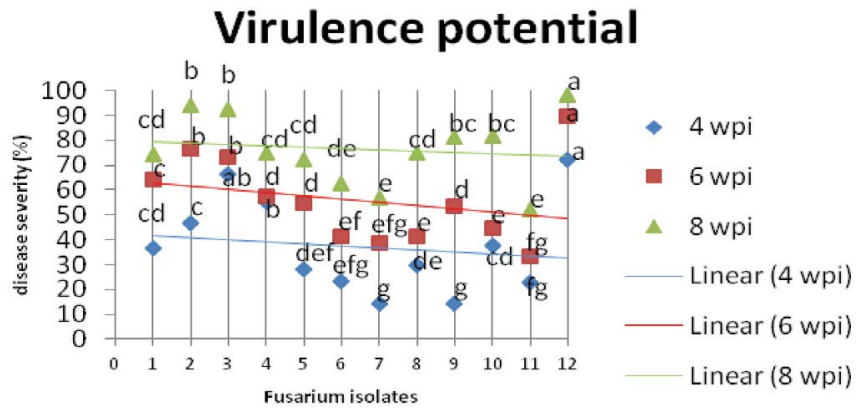

Figure 2: Severity of twelve different $F$. oxysporum isolates $(1: 12)$ recorded after 4 weeks ( 4 wpi), 6 weeks ( 6 wpi) and 8 weeks ( 8 wpi) on individual inoculated tomato plants. Means paired with different letter(s) are significantly differed based on Tukey's test $(p \leq 0,05 ; \mathrm{n}=5)$

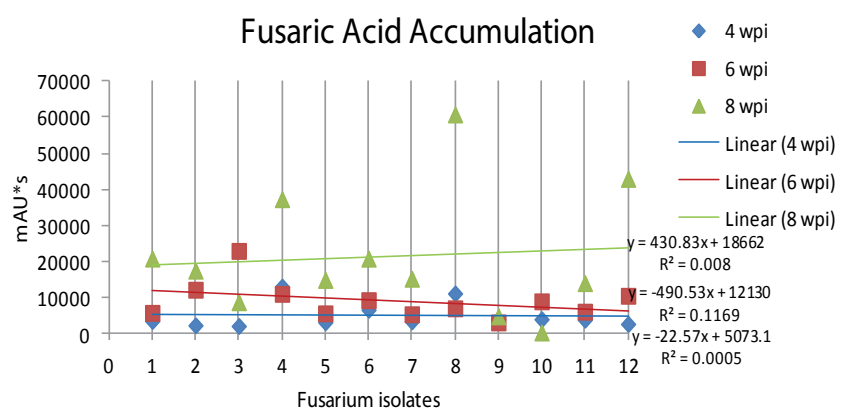

Figure 3: In vitro accumulation of Fusaric acid (FA) detected within twelve different $F$. oxysporum isolates (1:12) at 4 weeks ( 4 wpi), 6 weeks $(6$ wpi) and 8 weeks (8 wpi) post inoculation on HPLC (270 nm wavelength).

among the tested isolates with regard to FA production under in vitro conditions. Results of 4 weeks post inoculation demonstrated that the highest amounts of FA were 13000, 11101, 6559 and $3936 \mathrm{mAU}$ S of isolates 4, 8, 6 and 10 respectively (Figure 3) while the highest amounts of FA at 6 weeks post inoculation were 22926, 12136, 10991 and 10451 mAU*S, of isolates 3, 2, 4 and 12, respectively (Figure 3). On the other hand, the highest amounts of FA at 8 weeks post inoculation were 60816,42926 and $37275 \mathrm{mAU}{ }^{*}$ S of isolates 8,12 and 4 respectively (Figure 3).

The obtained results for both pathogenicity test and HPLC chromatogram analysis revealed that, no clear evidence for positive correlation could be detected between in vitro FA accumulation and severity of the tested Fusarium isolates toward tomato plants at 4 weeks post inoculation (Figure 4). Thus, the highest disease severity was recorded with isolates $12,3,4$ while only isolate 4 showed relative high ability to produce high concentration of FA comparing to the other isolates (Figure 4).

Six weeks after inoculation, the results illustrated in Figure 5 show that the most virulent isolates were $12,2,3$, and 4 which produced the highest concentrations of FA comparing to the other isolates.

Eight weeks post Fusarium inoculation, the results illustrated in Figure 6 show that among the most virulent isolates (12, 2, 3 and 8) only two isolates, 12 and 8 recorded relative high FA accumulation, while the other two isolates $(2,3)$ exhibited relative low FA producing capability comparing to other isolates.

Regarding to the accumulation of total secondary metabolites within the tested Fusarium isolates, the chromatogram analysis indicated that, the highest concentrations of total secondary metabolites was observed with isolates $8,4,7$ and 1, respectively (Figure 7) at four weeks post inoculation. At six weeks post inoculation, the highest concentrations of total secondary metabolites were 48654, 34517 and $26511 \mathrm{mAU}^{*} \mathrm{~S}$ which recorded with isolates 10,11 and 12 , respectively (Figure 7). The results obtained from HPLC analysis, at 8 weeks pos inoculation, showed that isolates $4,12,11$ and 1 produced the highest total secondary metabolites expressed as 91904, 89328, 49585, 42268 $\mathrm{mAU}$ S, respectively (Figure 7).

\section{Discussion}

Among all genera of plant pathogenic fungi that can infect tomato plants, Fusarium species are the most economically important to the production. Recently, in Egypt, the losses in tomato production due to Fusarium oxysporum attack reached up to 67\% [10]. Many Fusarium species are found to have the ability to produce toxic compound known as Fusaric acid (FA). FA is a natural mycotoxin produced by numerous Fusarium species under both in vitro and in vivo conditions $[3,16,18]$. Large concentrations of FA (greater than $10 \mathrm{M}$ ) were found to be extremely toxic to all living organisms including host plants, animals and human beings [4] while the least concentrations show antibiotic, insecticidal and pharmacological potentials $[19,20]$.

In the present study, the bioassay test was conducted under green house conditions to determine the pathogenicity and virulence potential of twelve Fusarium isolates belonging to F. oxysporum and to evaluate the evolution of Fusarium wilt disease by measuring the disease severity four, six and eight weeks after inoculation. Furthermore, the chemical analysis using High Pressure Liquid Chromatography (HPLC) was achieved to estimate the ability of the tested Fusarium isolates to produce Fusaric acid under in vitro conditions rather than to figure out the possible correlation between in vitro Fusaric acid accumulation and the Fusarium wilt disease development on tomato plants.

The obtained results from the bioassay demonstrated that all tested isolates were pathogenic toward the tomato plants and caused the typical symptoms corresponding to Fusarium wilt disease. Furthermore, significant differences were recorded among the tested isolates with regard to their virulence potential on tomato plants either four weeks, six weeks or eight weeks post inoculation. Four weeks after inoculation, the percentage of disease severity was ranged from $14.43 \%$ to $72.21 \%$. Meanwhile, the highest percentage of disease severity, $72.21 \%$, was observed with isolates 12 followed by isolate 3 which caused up to $66 \%$ of disease severity. Six weeks after inoculation, the disease severity was increased in general within all tested isolates. Remarkable, the highest percentage of disease severity $(89.50 \%)$ was observed again with isolate 12 followed by isolate $2(76.30 \%)$ and isolate $3(72.98 \%)$, respectively. Two weeks later, 8 weeks post inoculation, the disease severity reached up to $98 \%$ and this percentage was observed again with isolate 12 while isolates 2 and 3 caused the second (94.15\%) and the third (92.48\%) highest percentage of disease severity, respectively. These results demonstrated that isolates 12,2 and 3 were the most virulent isolates and therefore they caused the highest disease severity 4,6 or 8 weeks after the inoculation.

In the present study, the obtained results from HPLC chromatogram analysis revealed that all isolates were able to produce FA under the test conditions. Moreover, as observed before with disease severity and disease development, the chromatogram results showed that accumulation of FA was proportional positively within the incubation time with all tested isolates in general and in particular with isolates 1, 2, 5, 6, 7, 11 and 12. Thus the lowest FA accumulation 


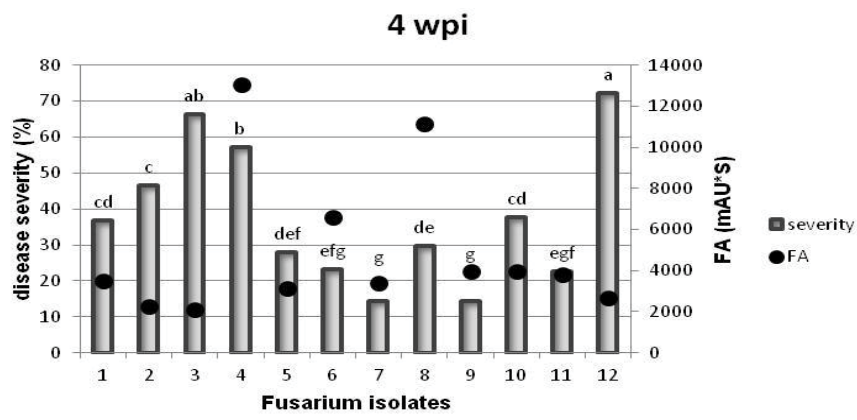

Figure 4: Correlation between virulence potential and Fusaric acid (FA) accumulation observed four weeks ( $4 \mathrm{wpi}$ ) after the individual inoculation of twelve different Fusarium oxysporum isolates (1:12). Means paired with different letter(s) are significantly differed based on Tukey's test $(p \leq 0,05 ; n=5)$.

\section{6 wpi}

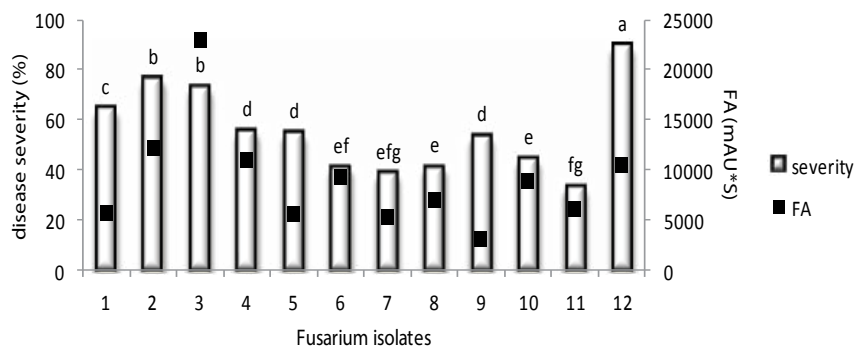

Figure 5: Correlation between virulence potential and Fusaric acid (FA) accumulation observed six weeks (6 wpi) after the individual inoculation of twelve $F$. oxysporum isolates (1:12). Means paired with different letter(s) are significantly differed based on Tukey's test $(p \leq 0,05 ; n=5)$.

within these isolates was detected 4 weeks after inoculation and the accumulation increased gradually to reach the highest level at eight weeks post inoculation. In contrary, isolates 3 and 10 recorded highest FA concentrations at six weeks post inoculation. For isolates 4 and 8 , the lowest FA concentrations were detected six weeks after inoculation. Indeed, no remarkable change with regard to FA accumulation was observed within isolate 9 .

Similar results were gained again from chromatogram analysis of the total secondary metabolites. Thus the results showed the accumulation of the total secondary metabolites was increased by increasing the incubation time with isolates $2,3,5,11$ and 12. In contrary, the production of total secondary metabolites was fluctuating with the rest of the isolates i.e., $1,4,6,7,8,9,10$. The results indicated also that some isolates produced exclusive compounds which were missed with the rest of the tested isolates. For example six weeks after inoculation, only isolate 5 induced the accumulation of the substance that detected at $17.3 \mathrm{~m}$ while isolates 6 and 7 only produced chemical substrate detected at retention time of $10.5 \mathrm{~m}$ (data no shown). Moreover, among all tested isolates only isolate 8 showed the ability to produce the metabolic substrate that can be detected at $270 \mathrm{~nm}$ wave length with retention time of 7.8 $\mathrm{m}$ (data not shown). Finally, the chemical assay showed also that isolate 12 produced relative high concentration $(9371 \mathrm{mAU}$ S) of the compound detected at $4.6 \mathrm{~m}$ (data not shown). These interesting observations make the isolation and the identification of these unknown detected compounds is quit important in the further studies to investigate their possible role in virulence potential and/ or the pathogenicity of Fusarium oxysporum isolates.
In general, the results obtained from the present study were in consistence with that of Bacon et al. [3] who screened 78 strains of Fusarium moniliforme, F. crookwellense, F. subglutinans, F. sambucinum, F. napiforme, F. heterosporum, F. oxysporum, F. solani, and $F$. proliferatum for their ability to produce fusaric acid, and found that no isolates of any of the Fusarium species examined were negative for the production of fusaric acid on autoclaved corn. Moreover, they reported that Fusarium oxysporum was observed to produce the maximum yield compared to other Fusarium species tested.

Since last century, FA was considered one of the first fungal metabolites implicated in the pathogenesis of tomato wilt symptoms caused by $F$. oxysporum f. sp. lycopersici $[1,11]$. Due to the using of primary and classical methods i.e., recording mycelial growth, these early studies had significant limitations and were not able through using these primary techniques to achieve the analytical rather than the quantitative determination of complicated substances as FA. Therefore nowadays using the advanced techniques i.e., HPLC, TLC, Mass spectroscopy, NMR and/or molecular markers for determining the virulence potential of Fusarium isolates has an increasing importance [15].

Paterson and Rutherford [21] investigated the relation between Fusaric acid and pathogenicity. They found that FA can cause the wilt symptoms on many host plants i.e., pepper, corn and vegetables through decreasing cell viability. Furthermore, Venter et al. [22] reported that FA responsible for potato tubers rot. More recently, several studies demonstrated that the high concentrations of FA affected the growth criteria and the essential physiological pathways in different living organisms including important economic plants through acting as

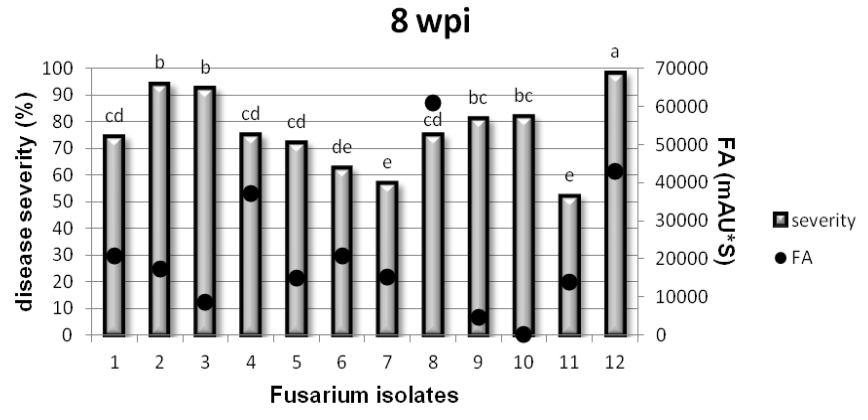

Figure 6: Correlation between virulence potential and Fusaric acid (FA) accumulation at eight weeks (8 wpi) after the individual inoculation of twelve $F$. oxysporum isolates (1:12). Means with different letter(s) are significantly differed based on Tukey's test $(p \leq 0,05 ; \mathrm{n}=5)$.

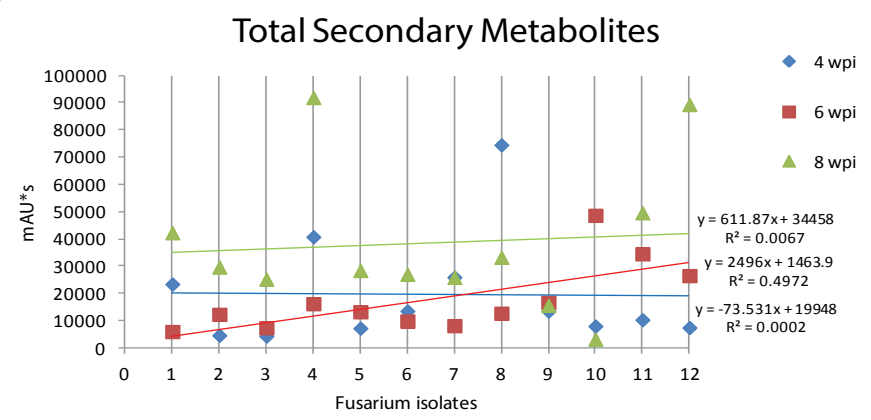

Figure 7: In vitro accumulation of total secondary metabolites recorded 4 weeks ( 4 wpi), 6 weeks $(6$ wpi) and 8 weeks $(8 \mathrm{wpi})$ post inoculation within twelve different $F$. oxysporum isolates (1:12) on HPLC at $270 \mathrm{~nm}$ wavelength. 
an enzyme inhibitor, dopamine agent and nucleic acid synthesis inhibitor. Indeed, FA was found to be able to chelate divalent cations, especially $\mathrm{Zn}$ and inactivate $\mathrm{Zn}$ finger proteins involved in DNA repair and protein synthesis $[19,23]$. In contrary, other studies mentioned that the relative low concentrations of FA could induce typical early defense response such as reactive $\mathrm{O}$ species 2 (ROS production) which involved in achieving biocontrol and nematicidal potential against some hazardous parasites as pine wood nematode Bursaphelenchus xylophilus [20].

\section{Conclusion}

In conclusion, the present study illustrate that the all tested Egyptian Fusarium oxysporum isolates are able to produce FA mycotoxin under the test conditions. It seems and for some extent, FA plays a distinctive role in the Fusarium wilt disease development on tomato plants during the middle and last stages of infection while this role is almost missing at the primary infection stage. Indeed, further studies focus on fractionation, purification and identification of some novel distinguish secondary metabolites which are accumulated within FA are highly needed to figure out whether FA alone or combined with other substances can be involved in the interactions between Fusarium oxysporum pathogenic isolates and their host plants.

\section{References}

1. Yabuta T, Kambe K, Hayashi T (1937) Biochemical studies of the 'bakanae' fungus of rice. I. Fusarinic acid, a new product of the 'Bakanae' fungus. J Agric Chem Soc Jpn 10: 1059-1068.

2. Malini S (1966) Heavy metal chelates of fusaric acid: in vitro spectrophotometry. Phytopathol Z 57: 221-231.

3. Bacon CW, Porter JK, Norred WP, Leslie JF (1996) Production of fusaric acid by Fusarium species. Appl Environ Microbiol 62: 4039-4043.

4. Eged S (2005) Thin-layer-chromatography-an appropriate method for Fusaric acid estimation. Biologia, Bratislava 60: 104.

5. Costa JM, Heuvelink E (2005) Introduction: The tomato crop and industry. In: Heuvelink E (Eds.). Tomatoes. CAB International, UK, pp: 1-19.

6. Sikora RA, Fernandez E (2005) Nematode parasites of vegetables. In: Luc M. Sikora RA, Bridge J (Eds.). Plant parasitic nematodes in subtropical and tropical agriculture. CABI Publishing: UK, pp: 319-392.

7. $\mathrm{CABI}$ (2011) Distribution Maps of Plant Diseases CABI, Wallingford, UK.

8. Armstrong GM, Armstrong JK (1981) Formae speciales and races of Fusarium oxysporum causing wilt diseases. In: PE Nelson, TA Toussoun, RJ Cook (eds.) Fusarium: Diseases, biology, and taxonomy. Pennsylvania State University Press, University Park and London, pp: 391-399.

9. Alabouvette C, Edel V, Lemanceau P, Olivain C, Recorbet G (2001) Diversity and interactions among strains of Fusarium oxysporum: Application and biological control. In: MJ Jeger and NJ Spence (eds.): Biotic interactions in Plant Pathogen Associations. CAB International, London, England, pp: 131-157.

10. Lasheen HHA (2009) Control of wilt disease of tomato through application natural compost and endo-mycorrhizal fungi. PhD Thesis, Plant Pathology Department, Faculty of Agriculture, Mansoura University, Egypt.

11. Gaumann E (1957) Fusaric acid as a wilt toxin. Phytopathology 47: 342-357

12. Hidaka H, Nagatsu T, Takeya K, Takeuchi T, Suda H (1969) Fusaric acid, a hypotensive agent produced by fungi. J Antibiot (Tokyo) 22: 228-230.

13. Porter JK, Voss KA, Bacon CW, Norred WP (1990) Effects of Fusarium moniliforme and corn associated with equine leukoencephalomalacia on rat neurotransmitters and metabolites. Proc Soc Exp Biol Med 194: 265-269.

14. Grattidge R, O'Brien RG (1982) Occurrence of third race of Fusarium wilt of tomatoes in Queensland. Plant Dis 66: 165-166.

15. Vakalounakis DJ, Fragkiadakis GA (1999) Genetic diversity of Fusarium oxysporum isolates from cucumber: differentiation by pathogenicity, vegetative compatibility and RAPD fingerprinting. Phytopathol 89: 161-168.
16. Notz R, Maurhofer M, Dubach H, Haas D, Défago G (2002) Fusaric acidproducing strains of Fusarium oxysporum alter 2,4-diacetylphloroglucino biosynthetic gene expression in Pseudomonas fluorescens $\mathrm{CHAO}$ in vitro and in the rhizosphere of wheat. Appl Envoron Microbiol 68: 2229-2235.

17. Plake BS, Kemmerer BE (1987) Statgraphics: Neil W. Polhemus and the Statistical Graphics Corporation. Rockville, MD: STSC. Computers in Human Behavior 3: 289-298.

18. Schouten A, van den Berg G, Edel-Hermann V, Steinberg C, Gautheron N et al (2004) Defense responses of Fusarium oxysporum to 2,4-diacetylphloroglucinol a broad-spectrum antibiotic produced by Pseudomonas fluorescens. Mol Plant Microbe Interact 17: 1201-1211.

19. Bouizgarne B, El-Maarouf-Bouteau H, Madiona K, Biligui B, Monestiez M, et al. (2006) A putative role for fusaric acid in biocontrol of the parasitic angiosperm Orobanche ramosa. Mol Plant Microbe Interact 19: 550-556.

20. Kwon HR, Son SW, Han HR, Choi GJ, Soo Jang K, et al. (2007) Nematicida Activity of Bikaverin and Fusaric Acid Isolated from Fusarium oxysporum against Pine Wood Nematode, Bursaphelenchus xylophilus. Plant Pathol J 23 318- 321.

21. Paterson RRM, Rutherford MA (1991) A simplified rapid technique for Fusaric acid detection in Fusarium strains. Mycopathologia 113: 171-173.

22. Venter SL, Steyn PJ, Steyn HSF (1996) Production of Fusaric acid by Fusarium oxysporum. Potato Research 39: 79-83.

23. van Rij ET, Girard G, Lugtenberg BJ, Bloemberg GV (2005) Influence of fusaric acid on phenazine-1-carboxamide synthesis and gene expression of Pseudomonas chlororaphis strain PCL1391. Microbiology 151: 2805-2814. 